\title{
Annual Report 1993
}

DOE grant FG03-292ER25128

Grant Title: Numerical and Asymptotic Studies of Complex Flow Dynamics

Principle Investigators: E. Coutsias, T. Hagstrom, J. Lorenz

\section{Technical Information}

Using analytical and numerical methods, we have investigated slightly compressible flows modeled by solutions of the Navier-Stokes equations [2,5]. General analytical results for ODEs and PDEs with highly oscillatory solutions have been obtained in collaboration with Prof. H. -0 . Kreiss [1,4]. Work has also been completed on the construction of boundary conditions at artificial boundaries for wave propagation problems and for parabolic systems $[9,10]$.

In the area of dynamical systems, we have analyzed a novel numerical approach to compute branches of invariant tori. A preliminary code has been developed [8]. We have obtained new results on the relationship between the true attractor and its numerical approximations for dissipative dynamical systems [6].

An efficient algorithm for the accurate solution of equations with polynomial coefficients has been developed and applications to the solution of the Navier-Stokes equations in disk geometry have begun $[11,12]$.

In most of these projects, students supported by the grant were involved. In the following, we give a more detailed description of our results.

\section{Slightly Compressible Flows}

Slightly compressible flows are described mathematically by solutions of the Navier-Stokes equations at low Mach number. The solutions vary on two different time scales, the slow convective scale describing an almost incompressible velocity and pressure field, and the fast acoustic scale describing sound waves. In mathematical terms, the sound waves are given by fast fluctuations of the pressure, the density, and the dilatation $\nabla \cdot u$ of the velocity field.

Supported by the grant, our student, Laura Dodson, has started work on a pseudospectral method for the direct numerical simulation of such flows, where the highly oscillatory part of the solution will be completely resolved. Two space dimensions and periodic boundary conditions are assumed.

\section{Highly Oscillatory Problems}

Slightly compressible flows are an example in which two time scales are present, the fast scale being highly oscillatory. Many other examples occur in oceanography and meteorology. Often one is only interested in the slow solutions or in the slow part of a general solution, which may be defined as a projection onto a slow manifold.

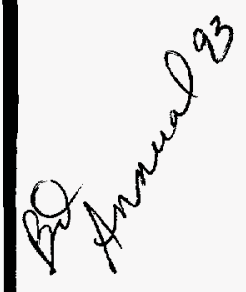




\section{DISCLAIMER}

Portions of this document may be illegible in electronic image products. Images are produced from the best available original document. 


\section{DISCLAIMER}

This report was prepared as an account of work sponsored by an agency of the United States Government. Neither the United States Government nor any agency thereof, nor any of their employees, makes any wartanty, express or implied, or assumes any legal liability or responsibility for the accuracy, completeness, or usefulness of any information, apparatus, product, or process disclosed, or represents that its use would not infringe privately owned rights. Reference herein to any specific commercial product, process, or service by trade name, trademark, manufacturer, or otherwise does not necessarily constitute or imply its endorsement, recom. mendation, or favoring by the United States Government or any agency thereof. The views and opinions of authors expressed herein do not necessarily state or reflect those of the United States Government or any agency thereof. 
In collaboration with Prof. H.-O. Kreiss, we have studied the existence of a slow manifold and have related its existence to the bounded derivative principle. Also, estimates on the interaction between the slow and the fast scale have been obtained $[1,4]$.

\section{Boundary Conditions at Artificial Boundaries}

A fundamental problem in the long time simulation of wave propagation (and other evolutionary processes) is the imposition of accurate "radiation" boundary conditions at artificial, computational boundaries. Our recent studies of this problem are published in $[2,5,9,10]$.

Under simplifying assumptions (linearization and localization), it is possible to represent exact boundary operators using Fourier-Laplace transformation. To derive useful approximations that are local in time and space, asymptotic analysis is applied. For the compressible NavierStokes equations, low Mach number approximations have turned out to be quite successful in comparison with conditions derived via energy estimates $[2,5]$.

\section{Dynamical Systems}

We have continued our collaboration with Prof. L. Dieci, Georgia Tech, on the numerical computation of invariant manifolds of dynamical systems. In particular, we have implemented a preliminary continuation code to follow a branch of invariant tori numerically. The tori deform and finally break as system parameters change.

Our approach is a numerical implementation of the Hadamard graph transform, a powerful analytical technique used in the perturbation theory of invariant manifolds (Fenichel, Indiana Univ. Math. J. 1971). Since our numerical approach mimics this analytical theory, we expect to be able to distinguish between analytical difficulties for continuation (torus breakdown) and numerical difficulties, such as insufficient resolution. An analysis of the numerical approach has been completed [8].

In related work, our student, Peter Kindilien, has implemented an algorithm to compute invariant curves of maps. Among others, applications to the Josephson junction and the van der Pol oscillator are under investigation.

Forced dissipative systems of ODEs and PDEs often have a universal attractor $\mathcal{A}$, which contains all non-transient states. If the system is studied numerically, the attractor $\mathcal{A}$ becomes replaced by a numerical attractor $\mathcal{A}_{h}$. General relations between $\mathcal{A}$ and $\mathcal{A}_{h}$ are studied in [6]; in particular, the Hausdorff dimensions of the true and the numerical attractor are compared.

\section{A Spectral Method for ODEs with Rational Coefficients}

The solution of the Poisson and Helmholtz equations in various separable geometries leads to BVPs for equations of the form $\sum_{k} p_{k}(x) D^{k} u=f(x)$. The solution of such equations by spectral methods offers great advantages in accuracy, but is complicated by the fact that the differential operators have full matrix representations in most spectral families (the only exceptions are Fourier and Hermite bases). For the functions that are most useful for the solution of two-point boundary value problems, namely Jacobi polynomials of various orders, 
the representation of differential operators leads to full, poorly conditioned upper triangular matrices. The conditioning problem is typically exacerbated by the inclusion of boundary conditions in the form of Lanczos tau constraints.

We have developed an algorithm that can solve the above BVPs efficiently, in terms of banded matrices whose bandwidth is of the order of the sum of the highest polynomial degree and the highest operator order present. The key idea is the observation that if the spectral family in question satisfies a simple type of recursion among polynomials and their derivatives (which includes the recursion for symmetric Jacobi polynomials), then integration, with a properly restricted range, becomes a banded operator. The ensuing efficient method leads, moreover, to well conditioned matrices as it completely avoids the need for tau conditions. The Chebyshev polynomials, the basis we are actually using, offer the added advantage of the FFT. Details will be published in $[11,12]$.

Supported by the grant, our student, David Torres, has studied applications to the solution of the incompressible Navier-Stokes equations in a disk geometry.

\section{Publications Resulting from the Award}

1. Kreiss, H.-O., Lorenz, J.: Manifolds of slow solutions for highly oscillatory problems. Indiana University Math. J. 42, 1169-1191 (1993).

2. Hagstrom, T., Lorenz, J.: Boundary conditions for models of slightly compressible flow. In: Applications of Advanced Computational Methods for Boundary and Interior Layers. J.J.H. Miller (editor), Boole Press, Dublin (1993).

3. Kloeden, P. E., Lorenz, J.: Lyapunov functions and the approximation of attractors. In: Nonlinear Dynamics: Attractor Approximation and Global Behaviour, pp. 93-98. N. Koksch, V. Reitmann, T. Riedrich (Editors), Technische Universität Dresden, 1993.

4. Kreiss, H.-O., Lorenz, J.: On the existence of slow manifolds for problems with different time scales. Phil. Trans. R. Soc. Lond. A, 346, 159-171 (1994).

5. Hagstrom, T., Lorenz, J.: Boundary conditions and the simulation of low Mach number flows. In: Environmental Acoustics, International Conference on Theoretical and Computational Acoustics, Vol. 2. D. Lee, M. H. Schultz (eds.), World Scientific, Singapore, 1994.

6. Lorenz, J.: Numerics of attractors and invariant manifolds. In: Chaotic Numerics (P. Kloeden, K. Palmer, eds.), AMS Series on Contemporary Mathematics 172 (1994), pp. $185-202$.

7. Lorenz, J., editor, Mathl. Comput. Modelling, Vol. 20, No. 10/11 (1994). Special Issue on Theory and Numerical Methods for Initial-Boundary Value Problems. 
8. Dieci, L., Lorenz, J.: Computation of invariant tori by the method of characteristics. SIAM J. Numer. Anal. Vol. 32, No. 5, 1436-1474 (1995).

9. Hagstrom, T., Hariharan, S.I., and R.C. MacCamy: On the accurate long-time solution of the wave equation in exterior domains. Math. Comp., 63, 507-539 (1994).

10. Hagstrom, T., Open boundary conditions for a parabolic system, Math. Comp. Mod., 20, 55-68 (1994).

11. Coutsias, E., Hagstrom, T., and Torres, D., An efficient spectral method for ordinary differential equations with rational function coefficients. Math. Comp., (1996), to appear.

12. Coutsias, E., Hagstrom, T., Hesthaven, J., and Torres, D., Integration preconditioners for differential operators in spectral $\tau$-methods. Houston J. Math., (1996), to appear.

\section{Education and Human Resources}

The following students were supported by the grant during the summer of 1993 .

\section{Laura Dodson, Graduate Research Associate}

Expertise and Role in the Project: Implementation of a pseudospectral method for solving the slightly compressible Navier-Stokes equations.

\section{Peter Kindilien, Graduate Research Associate}

Expertise and Role in the Project: Implementation of numerical methods for computing invariant manifolds of dynamical systems.

A result of this work was the Ph.D. thesis: Numerical Approximation of Invariant Curves and Invariant Tori of Systems of Ordinary Differential Equations completed in 1995.

\section{David Torres, Graduate Research Associate}

Expertise and Role in the Project: Development of a fast solver for Chebyshev spectral discretizations of general second order equations with low degree polynomial coefficients.

A result of this work was the Ph.D. thesis: Integration Operators in the Spectral $\tau-M e t h o d$ with Navier-Stokes Applications completed in 1995. 